\title{
Sprachbewusste Kartenarbeit? Beobachtungen zum Karteneinsatz im Geographieunterricht
}

\author{
“alexandra.budke@uni-koeln.de, Institut für Geographiedidaktik, Universität zu Köln \\ ** miriam.kuckuck@uni-osnabrueck.de, Geographiedidaktik am Institut für Geographie der Universität Osnabrück ( $\square$ korresp. Autorin) \\ ${ }^{* * *}$ m.morawski@uni-koeln.de, Institut für Geographiedidaktik, Universität zu Köln
}

eingereicht am: 08.11.2016, akzeptiert am: 04.10.2017

\begin{abstract}
Ein sprachbewusster Geographieunterricht wird aufgrund zunehmender sprachlicher Heterogenität in den Klassen notwendig. Die Reflexion sprachlicher Anforderungen und Methoden bei der Kartenarbeit ist ein wichtiger Baustein für die Umsetzung sprachbewussten Geographieunterrichts. In dem Artikel wird der Frage nachgegangen, inwiefern Kartenarbeit im Geographieunterricht tatsächlich sprachbewusst stattfindet. Dafür wurden 585 Geographiestunden in der Sekundarstufe I beobachtet und analysiert.
\end{abstract}

Keywords: Sprachbewusster Geographieunterricht, Kartenarbeit, Praxissemester

\section{Language awareness when working with maps? Observations concerning the usage of maps in the geography classroom}

Due to increasing linguistic heterogeneity in geography classes, language-aware geography education becomes crucial. The reflection of language requirements and methods when working with maps is a necessary means when performing languageaware geography lessons. This article assesses the question, to what extent working with maps happens with languageawareness. In that matter, 585 geography lessons have been monitored and analyzed.

Keywords: language awareness, language-aware geography education, maps

\section{Einleitung und Forschungsfrage}

Der Geographieunterricht hat besonderen Einfluss auf die Entwicklung der Schüler/innen zu mündigen Bürgerinnen und Bürgern, da er sich intensiv mit aktuellen gesellschaftlichen Problemen und deren Lösungsmöglichkeiten beschäftigt. Themen wie Migration oder nachhaltige Ressourcennutzung haben eine hohe Alltagsrelevanz für die Schüler/innen zu denen sie in der Regel viel Vorwissen mitbringen. Im Unterricht sollten die Schüler/innen Gelegenheit bekommen, sich kritisch mit den gesellschaftlichen Diskursen zu diesen Problemen auseinanderzusetzen, die Argumente der verschiedenen Interessensgruppen $\mathrm{zu}$ verstehen und $\mathrm{zu}$ bewerten sowie eine begründete eigene Meinung zu entwickeln.

Dabei sollte auch die Bedeutung von nicht-linearen Darstellungsformen wie Karten in gesellschaftlichen Diskursen thematisiert werden. Sehr häufig finden sich in den täglichen Pressemitteilungen Berichte, die mithilfe von Karten untermauert werden. Die Karten sollen die Argumente im Text belegen, unterstreichen und/oder die Leser/innen fesseln. Der kritische Umgang mit Medienberichten, die Karten enthalten, ist demnach ein wesentlicher Aspekt unserer Lebenswelt und sollte insbesondere im Fach Geographie geübt werden. Der Umgang mit kartenbasierten Diskursen stellt die Schüler/innen vor hohe inhaltliche, methodische und auch sprachliche Herausforderungen. Diesbezügliche Kompetenzen sollten sukzessive im Geographieunterricht aufgebaut werden.

Die durchgängige Sprachbildung der Schüler/innen in allen Unterrichtsfächern wird in verschiedenen Veröffentlichungen eingefordert (Gogolin et al. 2011; Becker-Mrotzek et al. 2013; Vollmer \& Thürmann 2013). Auch der Geographieunterricht sollte sprachbewusst erfolgen. Sprachbewusster Geographieunterricht ist in diesem Kontext ein Unterricht, der sich 
den sprachlichen Anforderungen bewusst wird, die von den Schülerinnen und Schülern bewältigt werden müssen, um geographische Fragestellungen zu beantworten und der diese Anforderungen flexibel vor dem Hintergrund der Schüler/innengruppe sprachlich reflektiert (u.a. Budke \& Weiss 2014: 14). Die geographiebezogene Sprachkompetenz meint damit nicht nur die Beherrschung der Fachsprache, sondern auch Fähigkeiten wie die Beschreibung verschiedenster (Geo-)Medien, ihre Analyse und Bewertung.

Die genauen sprachlichen Anforderungen des Geographieunterrichts und die tatsächliche sprachliche Förderung wurden allerdings bisher nur in Ansätzen definiert und empirisch erforscht. Durch diesen Artikel möchten wir zur Schließung dieser Forschungslücke beitragen und der Frage nachgehen, inwiefern die Kartenarbeit im Geographieunterricht tatsächlich sprachbewusst stattfindet. Folgende Teilfragen sind dabei von Interesse:

- Wie hoch sind die durch die Operatoren definierten sprachlichen Anforderungen bei von denen von den Lehrkräften eingesetzten Aufgaben bei der Kartenarbeit?

- Inwiefern findet sprachliche Förderung und Unterstützung der Schüler/innen bei der Kartenarbeit zur Überwindung von sprachlichen Hürden durch die Lehrkräfte statt?

- Inwiefern lassen sich jahrgangsbezogene Unterschiede in Umfang und Intensität der sprachlichenFörderungbeiderKartenarbeitbeobachten?

Die im Weiteren vorgestellten umfangreichen empirischen Daten beruhen auf 585 beobachteten Geographiestunden in Nordrhein-Westfalen und Niedersachsen.

Bevor sich der Artikel der empirischen Arbeit widmet, wird eine theoretische Annäherung stattfinden, die eine gehaltvolle Einordnung und Reflexion der Ergebnisse möglich macht.

\section{Bedeutung von Sprache im Geographie- unterricht}

Sprache hat - wie in jedem Unterricht - auch im Geographieunterricht eine Bedeutung als Lernmedium, als Lern- und Reflexionsgegenstand, als Kommunikationsmittel sowie als Mittel zur Leistungsüberprüfung (vgl. Michalak et al. 2015). Budke und Weiss (2014) identifizieren acht Ziele in ihrer Analyse sprachlichkommunikativer Anforderungen in deutschen Lehrplänen: (1) Informationsaufnahme aus GeomedienBeschreibung, (2) Analyse und Bewertung, (3) Erwerb und Anwendung des Fachwortschatzes, (4) Durchführung geographischer Befragungen, (5) Entschlüsselung gesellschaftlicher Diskurse, (6) Entwicklung und Durchführung von Diskussionen und Argumen- tationen, (7) Präsentationen von Ergebnissen und die (8) kritische Reflexion bezogen auf Material, Quellen und Lernweg. Bis auf den Punkt (4) sind alle Anforderungen auf die Kartenarbeit und die problemlösende Arbeit mit gesellschaftlichen Diskursen beziehbar. In den nationalen Bildungsstandards finden sich entsprechende Kompetenzen in den Kompetenzbereichen Fachwissen, Räumliche Orientierung und Kommunikation (vgl. DGfG 2014).

Das auf verschiedenen Erhebungen basierende hier verkürzt dargestellte GLAM - Geographic Language Model (GLAM) ermöglicht die Strukturierung sprachlicher Anforderungen und darauf bezogene sprachliche Hilfen im Geographieunterricht (Morawski \& Budke 2017a). Eine der Grundlagen des Modells ist der gemeinsame europäische Referenzrahmen für Sprachen (CEFR 2011: 14), der kommunikative Kompetenzen in den Bereichen Rezeption, Interaktion, Produktion, Mediation unterscheidet. Geographische Fachsprache wird im Modell von Bildungs- und Alltagssprache umschlossen (siehe Abb. 1). Besonders zentral ist für den Geographieunterricht die Ebene der Fachsprache. Diese wird nach Wort-, Satz- und Textebene differenziert.

Ebene E1 bis Ebene E3 visualisieren den Anstieg sprachlicher Anforderungen. Während E1 beispielsweise lediglich das Nennen von Begriffen umfasst, beinhaltet Ebene E3 komplexere Sprachhandlungen. Diese sind für das Partizipieren an gesellschaftlichen Diskursen und für die Reflexion der Mechanismen zur Teilkonstruktion dieser Diskurse nötig. Diese Handlungen beinhalten typische sprachliche Diskursformen im Geographieunterricht wie bspw. die Argumentation, Reflexion oder Beurteilung. In Ebene E3 kann beispielsweise die Aufgabe für die Schüler/innen sein, die Darstellungsweise und Aussage einer Karte zu bewerten.

Für die Durchführung von Handlungen auf Ebene E3 müssen die Schüler/innen sprachliche Handlungen und Fähigkeiten aus E1 und E2 beherrschen. Dafür sind die Verwendung sprachlicher Mittel im Sinne von reinen Nennungen von Fachbegriffen der Ebene E1 und basale Handlungen wie Beschreibungen und Zusammenfassungen nötig, wie auf E2 abgebildet. Für die geographische Diskursfähigkeit E3 benötigen die Schüler/innen somit in besonderer Weise weiterentwickelte rezeptive, interaktive, produktive sowie transferierende Fähigkeiten. Mit rezeptiven Kompetenzen kann bspw. die Dekodierung von u. a. sprachlichen Kartenelementen und Verbindung mit textlicher Information gemeint sein. Interaktive Fähigkeiten beinhalten z. B. die adressatengerechte Formulierung im Kontext einer kooperativen Gruppenarbeit, die im schulsprachigen Kontext eine Diskussion der Rheinnutzung umfasst, während produktive die eigenständige Versprachlichung von bspw. 


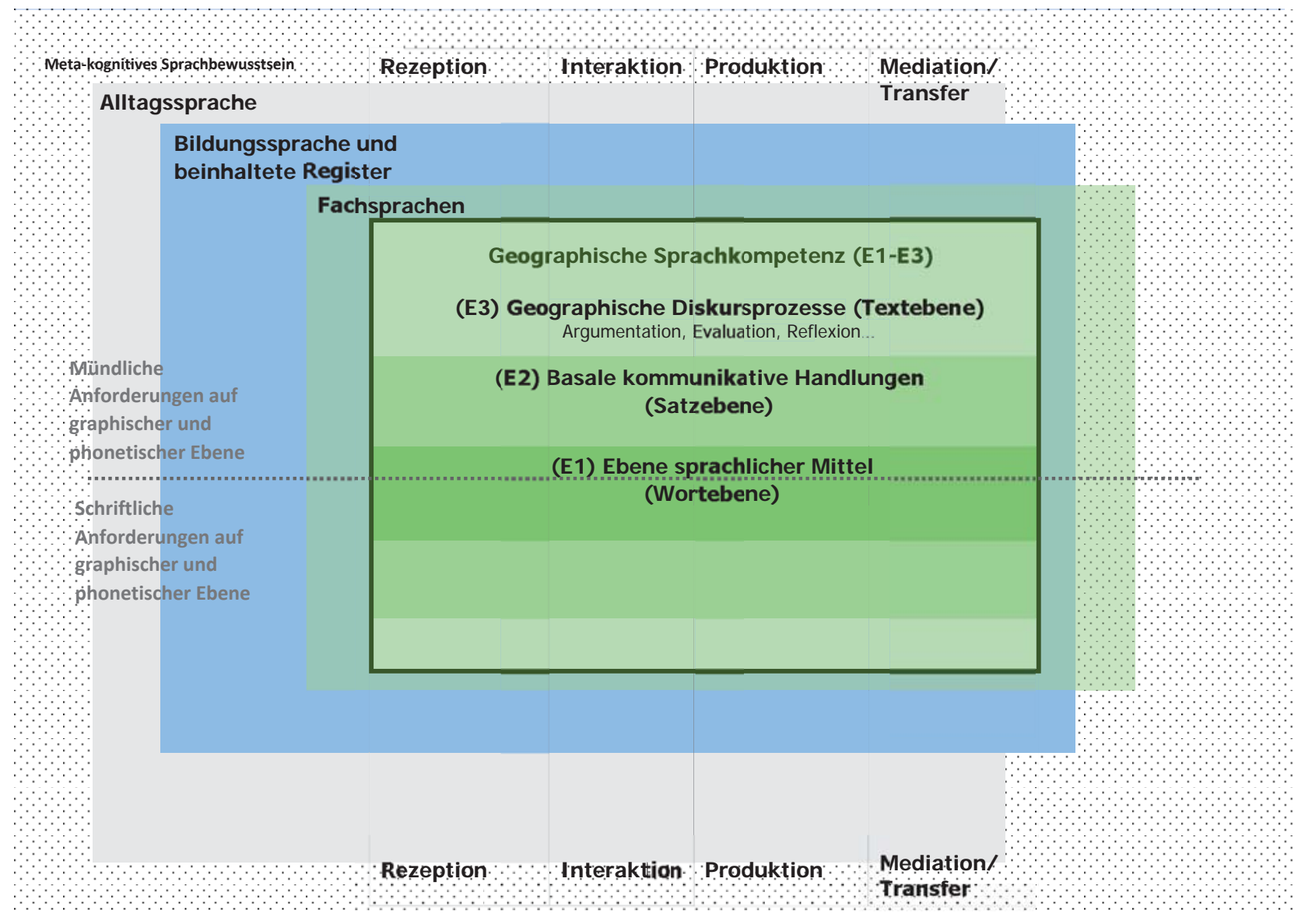

Abb. 1: Modell zur Strukturierung sprachlicher Anforderungen im Geographieunterricht (übersetzt und vereinfacht aus: Morawski \& Budke 2017a: 64)

Karteninhalten umfassen kann. Transferierende Fähigkeiten im Rahmen von Mediation beinhalten zum einen auf der Transferebene des Textsortenwechsels die Umwandlung der Informationen aus der Karte in einen mündlichen oder schriftlichen Text. Auf der anderen Seite bedeutet die Mediation eine Aufarbeitung des Textes dezidiert für Dritte, d.h. dass die Schüler/ innen somit eine vermittelnde Instanz einnehmen. Dieser progressive Aufbau der sprachlichen Anforderungen wird anhand des Beispiels Kartenarbeit nun vertiefend erläutert.

\section{Sprachliche Anforderungen bei der Arbeit mit Karten}

Die Bearbeitung von Karten, die als Quellen oder Belege für Argumentationen eingesetzt werden, ist abhängig von der im Unterricht gegebenen Aufgabenstellung, die eine Sprachhandlung einfordert. Diese Sprachhandlung steht im Zusammenhang mit den Operatoren, die als Signal- und Aufforderungswörter verstanden werden, welche die Handlung bestimmen. Die Bildungsstandards der Geographie teilen die Operatoren mit denen Aufgaben formuliert werden in drei Anforderungsbereiche ein: 1. Reproduktion (u. a.
Beschreiben), 2. Reorganisation und Transfer (u.a. Analysieren, Erklären) und 3. Reflexion und Problemlösung (u. a. Bewerten) (vgl. DGfG 2014: 32 f.). Dabei wird davon ausgegangen, dass die sprachlichen und inhaltlichen Anforderungen an die Schüler/innen mit den Anforderungsbereichen steigen.

Nach Hemmer et al. (2010) und dem Ludwigsburger Modell der Kartenauswertekompetenz setzt sich der verstehende Umgang mit Karten aus den Teilkompetenzen ,Karten zeichnen', ,Karten auswerten' und ,Reflexion über Karten'zusammen. Diese Bereichesind mit verschiedenen sprachlichen Anforderungen verknüpft.

\subsection{Karten auswerten}

\subsubsection{Karten lesen}

Der Bereich der Kartenauswertung gliedert sich in die Bereiche des Kartenlesens und des Karteninterpretierens (vgl. Hemmer et al. 2010). Zum Bereich des Kartenlesens zählen zum einen die grundlegende Handlung des Dekodierens des sprachlichen, symbolisierten, geometrischen und generalisierten Codes der Karte sowie zum anderen die Handlung des Kartenbeschreibens. Der sprachlich-textuelle Code, der auf einer Karte zu finden ist, beschränkt sich zunächst auf 
Nomen und nominale Phrasen, deren Erfassung den Handlungen in den Bereichen E1 und E2 des Modells zuzuordnen ist (siehe Abb. 1). Dazu gehören bspw. die Benennung von topographischen Gegebenheiten wie Städten oder Gewässern sowie die Erfassungen der Signaturen. Das Vorwissen der Schüler/innen und die kognitiven Schemata, die sie anwenden können, um das in der Karte durch Farben und Formen enthaltene Wissen zu entschlüsseln, ist entscheidend (vgl. Schnotz \& Dutke 2004; Schnotz 2002).

Vollmer (2011) fasst zentrale, sprachliche Diskursfunktionen zusammen, die seiner Analyse nach in allen Fächern und über Fächer hinweg eine Rolle spielen. Beschreibung von fachunterrichtlich relevanten Prozessen und Ereignissen beziehen sich nach Vollmer (2011) auf „Tatsachen“, die offensichtlich oder zumindest für andere nachvollziehbar sind und sich jeglichen eigenen Urteils enthalten. Eine beschreibende Sprache stellt bereits erhebliche Anforderungen an den Wortschatz, vor allem was Begriffe zur qualitativen und quantitativen Differenzierung betrifft (vgl. ebd.). Sie ist die Grundlage für weitergehende Analysen und Bewertungen. Es muss die Frage gestellt werden, wie sich diese Ausschärfung in der Fachdidaktik Geographie darstellt und somit welche Anforderungsebenen, z. B. auf textkohärenter und lexikalischer Ebene die Funktion Beschreibung im Geographieunterricht im Kontext der Karte stellt. Weiter ist zu klären, wie die Beschreibung als basale sprachliche Handlung für weitere Handlungen in diesem Kontext zu verstehen ist.

Gerade die von Vollmer (2011) angesprochene Enthaltung von Urteilen, ist ein Kriterium für Beschreibungen im Geographieunterricht. Hemmer et al. (2010) verstehen darunter eine Handlung, welche die Schüler/innen auffordert, die primären karteninternen Informationen bezüglich der Raumstrukturen und der Einzelphänomene zu nutzen und zu erfassen (vgl. Hemmer et al. 2010; Lenz 2005).

Die Identifikation der für die Beschreibung notwendigen Kartenelemente und deren Dekodierung stellt eine schwierige koordinative Aufgabe dar. Visuell-räumliche Beziehungen müssen von den Schülerinnen und Schülern auf semantische Beziehungen übertragen werden, um so die bildliche Darstellung in Sprache zu übersetzen (vgl. Ullrich et al. 2012). Gerade die Übertragung des sprachlichen Codes der Karte in die Form eines mündlichen oder schriftlichen Textes, wie bspw. einer Beschreibung, kann Schüler/innen mit geringeren Deutschfähigkeiten größere Schwierigkeiten bereiten (vgl. Wiegand 2006; Kölzer et al. 2015).

\subsubsection{Karten interpretieren}

Die Karteninterpretation setzt sich nach Hemmer aus den Handlungen ,Erklären` und ,Beurteilen` zusam- men. Die Durchführung von Analysen (Operatoren des Anforderungsbereichs II) erscheinen grundlegend für die Lösung von Beurteilungsaufgaben (Operatoren im Anforderungsbereich III). Der große Unterschied zum Kartenlesen besteht darin, dass bei einer Analyse nun externe Informationen zusätzlich zu den kartenimmanenten hinzugezogen werden sollen (Hemmer et al. 2010). Im Gegensatz zur Beschreibung müssen in einer Erklärung laut Vollmer (2011) die Ursachen von Ereignissen, Situationen oder Prozessen genannt werden. Diese können eingetreten sein oder noch in der Zukunft liegen.

Werden kartenbasierte Erklärungen angefertigt, müssen demnach Ursachen der in der Karte dargestellten Phänomene sowie Prozesse berücksichtigt werden. Durch die Integration externer Informationen und der Reorganisation entstehen komplexere sprachliche Darstellungen.

Verglichen zur den Funktionen ,Beschreiben' und ,Erklären'werden Sachverhalte und ihre Ursachen beim Beurteilen vor dem Hintergrund eines normativen Bezugssystems betrachtet und begründete Stellungnahmen werden abgegeben (vgl. Vollmer 2011). Eine kritische Beurteilungsfähigkeit von Karten (E3 in Abb. 1) ist nur dann erreichbar, wenn der Prozess der kartographisch codierten Wiedergabe der Wirklichkeit und deren gedanklichen Umsetzung in die entsprechende Raumvorstellung anhand vorheriger durchgeführter Handlungen und Funktionen verstanden wurde.

\subsection{Kartenreflexion}

Informationen in Karten werden durch bewusste Auswahl von Signaturen und Farben sowie Raumprojektionen durch den Kartenproduzenten komprimiert und besonders in Szene gesetzt. Die intensive, sprachliche Entschlüsselung, Beschreibung, Erklärung und Beurteilung des textuellen und piktoralen Codes (Signaturen) ist für die reflexive Kartenarbeit und die Reflexion von Karten in gesellschaftlichen Diskursen essentiell (vgl. Weidenmann 2004; Gryl et al. 2010). Als Diskurs versteht Foucault „die Gesamtheit von Regeln, die einer Praxis immanent sind“ (1973: 70). Durch alltägliche Kommunikation werden diese Praktiken und Regeln, bezogen auf den Raum, der im Geographieunterricht im Zentrum steht, neu ausgehandelt und reproduziert. Räume sind sozial konstruiert und können dadurch bestimmte (negative wie positive) Zuweisungen erhalten, was für eine Region konkrete Folgen haben kann. Die Kartenanalyse kann dabei neben der Kartographie als eigener Diskurs, als Bestandteil eines gesellschaftlichen Diskurses gesehen werden (vgl. Werlen 1997; Bauriedl 2007). Den Schülerinnen und Schülern sollte durch die Thematisierung im Unterricht verständlich gemacht werden, dass Karten 
immer Teil eines gesellschaftlichen Diskurses sind und Karten als Beleg in einer Argumentation angeführt werden können. Sie sind keine objektiven Wahrheiten, sondern müssen als soziale Konstrukte behandelt werden. Sie können im Unterricht dekonstruiert werden, was zur Förderung der kritischen Kartenlesekompetenz der Schüler/innen beitragen kann. Schritte bei der Dekonstruktion sind: Reflexion und offene Darlegung der Kartenproduzentinnen und Kartenproduzenten sowie der Absicht und Interessen der Kartennutzer/innen und der Codierungselemente (Mose \& Strüver 2009: 315; Gryl \& Kanwischer 2011). Diese Fähigkeit ist als Voraussetzung zur Teilnahme an gesellschaftlichen Diskursen zu verstehen und kann unter dem Begriff critical thinking oder im internationalen Gebrauch auch unter den high order thinking skills gefunden werden. Sprachlich und inhaltlich stellt die Kartenreflexion große Anforderungen an die Schüler/ innen, da die Karte im Kontext anderer Medien, welche den gesellschaftlichen Diskurs offenlegen, analysiert und bewertet werden muss.

\section{Forschungsdesign/Methodik}

Um zu untersuchen, inwiefern bei der Kartenarbeit im Geographieunterricht sprachliche Förderung der Schüler/innen stattfindet, wurde ein standardisierter Beobachtungsbogen entwickelt. Beobachtungen von Unterrichtseinheiten sind in der pädagogischen und didaktischen Forschung sowie in der Lehrer/innenausbildung eine wesentliche Erhebungsmethode, um empirisch belegbare Informationen über den Unterricht zu erheben. Beobachtungen gelten als ,harte Forschungsmethode, da sie eine gezielte, bewusste und methodisch kontrollierte Wahrnehmung und Dokumentation ermöglichen (vgl. Roos \& Leutwyler 2011: 192f.). Die von uns eingesetzte Fremdbeobachtung ist theorie- und kriteriengeleitet und daher strukturiert bzw. standardisiert. Des Weiteren kann diese Beobachtung den offenen Beobachtungen zugeordnet werden, da die Beobachtenden als solche deklariert und sichtbar im Klassenraum sitzen, jedoch nicht teilnehmend sind, da sie nicht direkt in das Unterrichtsgeschehen eingreifen (vgl. Diegmann 2013).

In unserer Untersuchung wurden 18 geschulte Studierende als Beobachter/innen eingesetzt, die während ihres Praxissemesters oder im Rahmen von Masterarbeiten an verschiedenen Schulen in Nordrhein-Westfalen und Niedersachsen Daten erhoben haben. Insgesamt wurden 585 Unterrichtsstunden beobachtet, wovon 29 Stunden (5\%) an Sekundarschulen, 35 Stunden (6\%) an Hauptschulen, 92 Stunden (15,7\%) an Gymnasien und 429 Stunden $(73,3 \%)$ an Realschulen beobachtet wurden. Dabei wurden die Klassenstufen 5 bis 11 beobachtet. Der Beobachtungsbogen wurde mit den Studierenden gemeinsam erarbeitet. Des Weiteren wurde durch Pretests überprüft, ob sich die für uns relevanten Merkmale des Unterrichts durch die standardisierten Kategorien des Erhebungsbogens abbilden lassen. Da die Erhebungen im Rahmen von Masterarbeiten bzw. des Praxissemesters stattfanden, war der Aufenthalt der Beobachter/innen sowohl für die Lehrer/innen als auch für die Schüler/innen keine Besonderheit. Die Lehrpersonen, die den Unterricht geführt haben, wussten, dass sie zur Kartenarbeit beobachtet wurden. Dies hat womöglich dazu geführt, dass häufiger mit Karten gearbeitet wurde als im ,normalen Unterricht. Einen Einfluss auf die Sprachförderung im Kontext der Kartenarbeit ist durch die Erhebung nicht entstanden, da den Lehrpersonen dieser spezielle Fokus nicht bekannt war.

Neben Informationen zur Schule und zur Lehrperson (z. B. Alter), waren für unsere Erhebung folgende allgemeinen Items von Bedeutung: Es wurde festgehalten, um welche Schulform es sich handelt (z.B. Realschule oder Gymnasium), in welcher Klasse beobachtet wird und ob es sich um das Fach Erdkunde oder Gesellschaftslehre handelt. Diese drei Items sind für die Auswertung in Bezug auf unsere Fragestellung wichtig. Nur wenn in der beobachteten Stunde eine Karte zum Einsatz kam, wurde der Erhebungsbogen weiter ausgefüllt. Von den insgesamt 585 beobachteten Stunden wurden in mehr als der Hälfte der Stunden (328 Stunden, 56,1\%) Karten eingesetzt (siehe Abb. 2). Des Weiteren wurden die Dauer des Karteneinsatzes, die Art der Karte, das Thema der Karte sowie die Darbietungsform erhoben.

Ferner wurden die Operatoren bzw. Fragewörter der Aufgabenstellung erhoben. Dazu wurden die Operatoren den drei bekannten Anforderungsbereichen gemäß der Nationalen Bildungsstands für das Fach Geographie (vgl. DGfG 2014) zugeordnet. Operatoren, die keinem dieser drei Anforderungsbereiche entsprechen, wie z. B. ausschneiden, kleben etc., wurden dem Bereich ,Grundanforderungen' zugeteilt, da es sich hierbei nicht um Operatoren zum Kompetenzerwerb handelt, sondern um Arbeitsaufforderungen (vgl. Budke et al. 2016.), die häufig in Kombination mit Dekodierungsaufgaben von Karten auftreten können. Plepis (2013: 22) unterscheidet für den Bereich des Lesens von Karten anhand des Ludwigsburger Modells der Kartenauswertekompetenz (vgl. Hemmer et al. 2010) die Bereiche Dekodieren, Beschreiben, Erklären und Beurteilen. Das Dekodieren gilt als Bereich der Kartenkompetenz, der das Wissen um Gestaltungsmittel und -methoden umfasst und sich nicht den drei Anforderungsbereichen zuordnen lässt. Er beinhaltet zum einen die symbolische 
Dekodierung (z. B. die Arbeit mit der Legende) und zum anderen die geometrische Dekodierung (z.B. Maßstab, Verebnung). Da aber in den von uns untersuchten Geographiestunden Arbeitsanweisungen genutzt wurden, die weder den drei Anforderungsbereichen noch dem Bereich des Dekodierens zugeordnet werden konnten, sondern darüber hinaus viel allgemeiner sind (z. B. ,kleben'), wurde die Kategorie ,Grundanforderungen' eingeführt. Diese umschießt sowohl das Dekodieren, als auch alle Arbeitsanweisungen, welche nicht durch Sprache ausgeführt werden müssen und von ihrer inhaltlichen Herausforderung unter denen des Anforderungsbereichs I liegen.

Ein weiterer wesentlicher Bestandteil der Beobachtung waren die sprachlichen Hilfen, die im Zusammenhang mit dem Einsatz der Karte im Unterricht erfolgten. Da jedoch nicht alle Studierenden den Einsatz von sprachlichen Hilfen bei der Kartenarbeit beobachteten, stehen zur Analyse dieses Aspektes nur die Daten von 376 Karten zur Verfügung, die im Unterricht eingesetzt wurden. Es wurden zehn Kategorien entwickelt, die im Erhebungsbogen mit den Skalen mündlich, schriftlich oder keine Hilfen angekreuzt werden konnten. Mit der Kategorie In ganzen Sätzen zu sprechen ist gemeint, dass die Lehrkraft die Schüler/innen auffordert, in ganzen Sätzen zu antworten. Damit werden bildungssprachliche Anforderungen deutlich gemacht und typische Muster typischer Satzproduktion im Mündlichen eingeübt. Dieser Aufforderung können die Schüler/innen allerdings nur nachkommen, wenn sie über das entsprechende sprachliche Wissen schon verfügen. Mit Fachvokabular (Michalak et al. 2015: 144) ist gemeint, dass die Schüler/innen von den Lehrkräften angehalten werden, Fachbegriffe zu nutzen. Die Lehrkraft macht damit die fachsprachlichen Anforderungen deutlich und gibt den Schülerinnen und Schülern die Möglichkeit einer Überarbeitung ihrer Äußerung. Dies ist allerdings nur möglich, wenn diese schon über die jeweiligen Fachbegriffe verfügen. Die von der Lehrkraft im Sinne eines Scaffolding genutzten sprachlichen Hilfen (vgl. Michalak et al. 2015: 161) in Form von Satzanfängen oder ganzen Satzvorgaben (vgl. Kniffka \& Neuer 2008) waren ebenfalls Kategorien, ebenso wie die Benutzung einer anderen Sprache, um den Schülerinnen und Schülern das Problem in ihrer Muttersprache zu erläutern. Als weitere Kategorie gelten die Umformulierungen seitens der Lehrperson oder die Aufforderung, Aussagen weiter zu präzisieren (vgl. Michalak et al. 2015: 144). Die Lehrperson gibt durch Umformulierungen sprachliche Vorbilder, an denen sich die Schüler/innen orientieren können. Auch die Erstellung eines Glossars in der Stunde (vgl. ebd.), die Aufforderung, lauter und deutlicher zu sprechen, sowie Nachfragen (vgl. ebd.: 155) wurden als sprachliche Hilfen aufgefasst und erhoben. Wenn Schüler/innen aufgefordert werden lauter und deutlicher zu sprechen, wird ihnen dabei geholfen, ihre Äußerungen in der Klasse verständlich zu machen und an der mündlichen Kommunikation teilnehmen zu können. Zudem kann ihr Selbstvertrauen gestärkt werden. Die genutzten Kategorien wurden deduktiv aus der Theorie abgeleitet. Dabei wurde sich nicht nur der theoretischen Auseinandersetzung innerhalb der Geographiedidaktik bedient, sondern vielmehr auch Fachliteratur aus dem Bereich ,Deutsch als Zweit- oder Fremdsprache' herangezogen.

Die Erhebungsdaten konnten diesen deduktiv gebildeten Kategorien zugeordnet und im Anschluss mit Hilfe der deskriptiven Statistik ausgewertet werden. Die Auswertung erfolgte mit dem Programm SPSS.

Beobachtungen als Forschungsinstrument weisen trotz ihrer Vorteile einige Nachteile auf. Denn sie können nur darüber Aufschluss geben, was, wie und wie häufig im Unterricht aufgetreten ist. Die Intentionen der Lehrer/innen, in den Stunden Karten einzusetzen und sprachliche Hilfen zu geben, konnte im Rahmen dieser Erhebung nicht erforscht werden (vgl. Roos \& Leutwyler 2011: 195). Trotz ihrer Vorstrukturierung und Transparenz sind Beobachtungsbögen nicht vollständig objektiv, denn es kann nur das beobachtet werden, was vorab Einzug in den Bogen erhalten hat (Mattisek et al. 2013: 67). Ferner weisen diverse Studien darauf hin, dass Beobachtungen aufgrund der subjektiven Wahrnehmung immer selektiv sind und immer mehrere Realitäten aufzeigen (vgl. Schnell et al. 2014; Bortz \& Döring 2015). Trotz der Standardisierung ist es demnach möglich, dass die verschiedenen Studierenden den Einsatz der Karte oder die sprachlichen Hilfen unterschiedlich bewerten und damit zu anderen Eintragungen kommen.

\section{Ergebnisse}

\subsection{Ergebnisse zum Karteneinsatz}

Zunächst wurde untersucht, in wie vielen der beobachteten Unterrichtsstunden Karten eingesetzt werden. Von den insgesamt 585 beobachteten Stunden wurden in mehr als der Hälfte der Stunden (328 Stunden, 56,1\%) Karten eingesetzt. Neben der Dauer des Karteneinsatzes, die Art der Karte, das Thema der Karte wurde die Darbietungsform erhoben. Innerhalb der 328 Unterrichtsstunden $(56,1 \%)$ wurden zum Teil mehrere und/oder verschiedene Karten eingesetzt. Insgesamt wurden 459 Karten genutzt. Davon waren 45,5\% thematische Karten, 29,2\% physische Karten, $15,9 \%$ topographische Karten und 9,4\% sonstige Karten. Mit 34\% sind der Atlas und das Schulbuch 
(27,7\%) die häufigsten Quellen der Karten (siehe Abb. 2). Nur $5 \%$ der im Unterricht eingesetzten Karten waren digital.

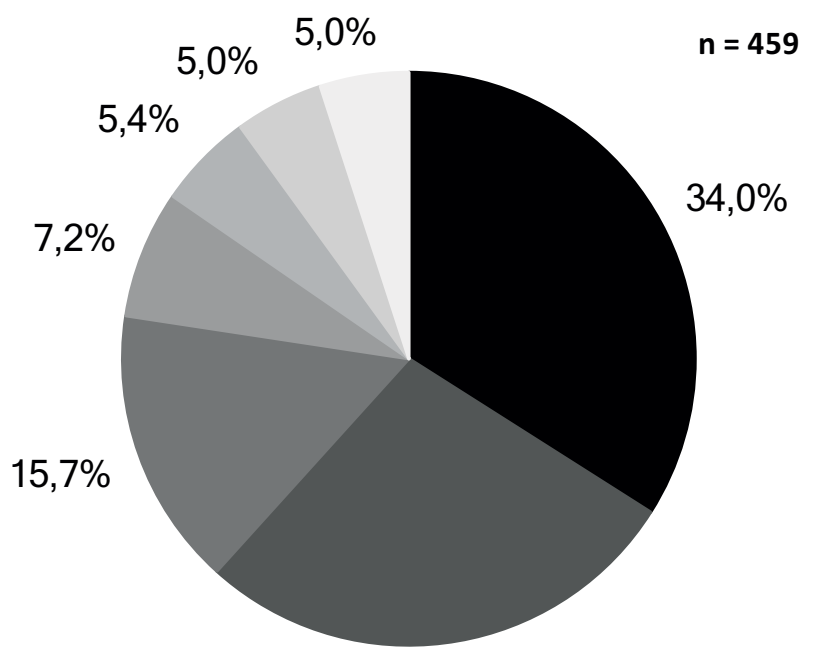

$27,7 \%$

Atlas Schulbuch Arbeitsblatt OHP Wandkarte Digital Sonstige

Abb. 2: Darbietungsformen von Karten in den beobachteten Unterrichtsstunden. Die Gesamtzahl $n=459$ entspricht der Anzahl der eingesetzten Karten (eigene Darstellung)

\subsection{Sprachliche und inhaltliche Anforderungen bei der Kartenarbeit}

Zunächst wurde untersucht, welche Aufgabenarten bei der Kartenarbeit eingesetzt wurden und welche inhaltlichen und sprachlichen Anforderungen sie an die Schüler/innen stellen. Dazu wurden die Operatoren der verwendeten Aufgaben erhoben und den Anforderungsniveaus der Bildungsstandards entsprechend kategorisiert (vgl. DGfG 2014). Die Analyse der Operatoren kann darüber Auskunft geben, mit welchem inhaltlichen und sprachlichen Anspruch Kartenarbeit im Geographieunterricht betrieben wird und in welchem Bereich verstärkte Förderung stattfindet bzw. welcher vernachlässigt wird. Es zeigt sich, dass 12,5\% der Aufgaben in den Grundanforderungsbereich entfallen. Hier werden die Operatoren ,suche und ,zeige' verwendet, die keine sprachlichen Anforderungen und nur sehr einfache inhaltliche Herausforderungen im Bereich des Dekodierens von Karten an die Schüler/innen stellen. Der Großteil der verwendeten Aufgabenfällt in den Anforderungsbereich I (74,7\%) (siehe Abb. 3). Hier werden besonders oft die Operatoren ,beschreibe oder ,nenne' sowie das Fragewort ,wo' verwendet (siehe Abb. 4). Ein Großteil der Aufgaben fördert demnach basale Kartenkompetenzen, die zum Dekodieren von Karten und zum Heraussuchen einzelner Informationen nötig sind. Dabei sind auch die sprachlichen Anforderungen gering, da nur einzelne (Fach-)Wörter, wie Ortsbezeichnungen und Himmelsrichtungen sowie basale Sprachhandlungen auf Satzebene erforderlich sind. Damit wird vorrangig das Lesen von Karten gefördert (siehe Kapitel 3). $11,8 \%$ der Aufgaben werden mit Operatoren formuliert, welche dem Anforderungsbereich II zuzuordnen sind. Es wird vorwiegend der Operator ,erkläre' verwendet. Durch die zugehörigen Aufgaben kann die geographische Diskursfähigkeit zur Erklärung von Sachverhalten gefördert werden. Aufgaben, in denen die Schüler/innen zu geographischen Fragestellungen kartenbasiert argumentieren, bewerten oder Stellung nehmen müssen, werden in den von uns beobachteten Geographiestunden äußerst selten gestellt (1\%). In absoluten Zahlen werden nur in drei Aufgaben von 288 Operatoren des Anforderungsbereichs III genutzt. Während die Zahl der Aufgaben, die im Grundanforderungsbereich und im Anforderungsbereich I gestellt werden, von der 5 . bis zur 10 . Klasse abnimmt und die Zahl der Aufgaben im Anforderungsbereich II in der gleichen Zeit zunimmt, was dem steigenden Leistungsniveau der Schüler/innen entspricht, findet im Anforderungsbereich III keine Veränderung und damit keine Progression statt. Wichtige geographische Diskursfähigkeiten wie die kartenbezogene Argumentation und Bewertung werden damit in der gesamten Sekundarstufe I offensichtlich nur wenig gefördert. Es zeigt sich bezüglich der Anforderungsbereiche der verwendeten Operatoren auch kein Unterschied zwischen jüngeren und älteren Lehrkräften. ${ }^{1}$

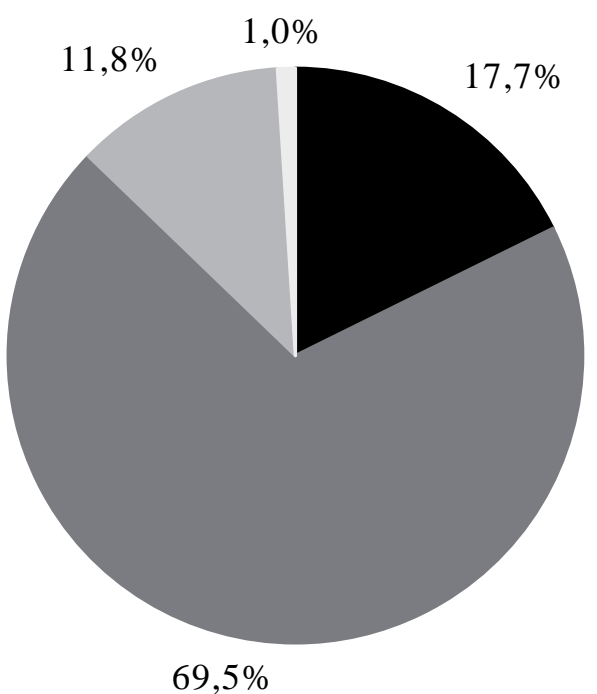

$n=288$

GA AFB I AFB II AFB III

Abb. 3: Anforderungen der Operatoren in kartenbezogenen Aufgabenstellungen im Unterricht. Die Gesamtzahl $n=288$ entspricht der Anzahl der Operatoren in Aufgaben, die klar einem Anforderungsbereich zugeordnet werden konnten ( $G A=$ Grundanforderungsbereich, $A F B=$ Anforderungsbereich, eigene Darstellung).

Jüngere Lehrkraft: bis 49 Jahre; ältere Lehrkraft ab 50 Jahren. 
Damit bestätigen sich Ergebnisse von Lindau (2012), welche die Umsetzung der ,räumlichen Orientierung' in Geographiestunden der Sekundarstufe I und II beobachtet hat. Auch in dieser Untersuchung dominiert der Anforderungsbereich $\mathrm{I}^{2}$ und der Anforderungsbereich III ist in allen beobachteten Klassenstufen bedeutungslos (neun von 365 Beobachtungen). Die Ergebnisse entsprechen zudem der Schulbuchuntersuchung von Budke (2011) und Budke et al. (2016), die zeigen, dass Aufgaben des Anforderungsbereichs III auch in Geographieschulbüchern nur sehr selten integriert sind. Nur 5\% aller Aufgaben zur Kartenarbeit in den analysierten Hauptschulbänden und nur $8 \%$ jener in den untersuchten Gymnasialbänden aus Nordrhein-Westfalen und Bayern ließen sich dem Anforderungsbereich III zuordnen (vgl. Budke et al. 2016). Ein Teil der Ergebnisse der Unterrichtsbeobachtungen könnte sich demnach durch die Aufgabenstellungen in Geographieschulbüchern erklären, da ca. ein Drittel der Karten, welche in dem von uns beobachteten Unterricht verwendet wurden, aus Schulbüchern stammten (siehe Abb. 2). Es zeigte sich jedoch bei anderen Darbietungsformen wie Atlanten oder Wandkarten, bei denen die Lehrer/innen Aufgaben eigenständig formulierten, auch kein höherer Anteil von Aufgaben im Anforderungsbereich III.

\subsection{Sprachliche Förderung bei der Kartenarbeit}

Es stellt sich die Frage, inwiefern die Lehrkräfte die sprachlichen Fähigkeiten ihrer Schüler/innen bei der

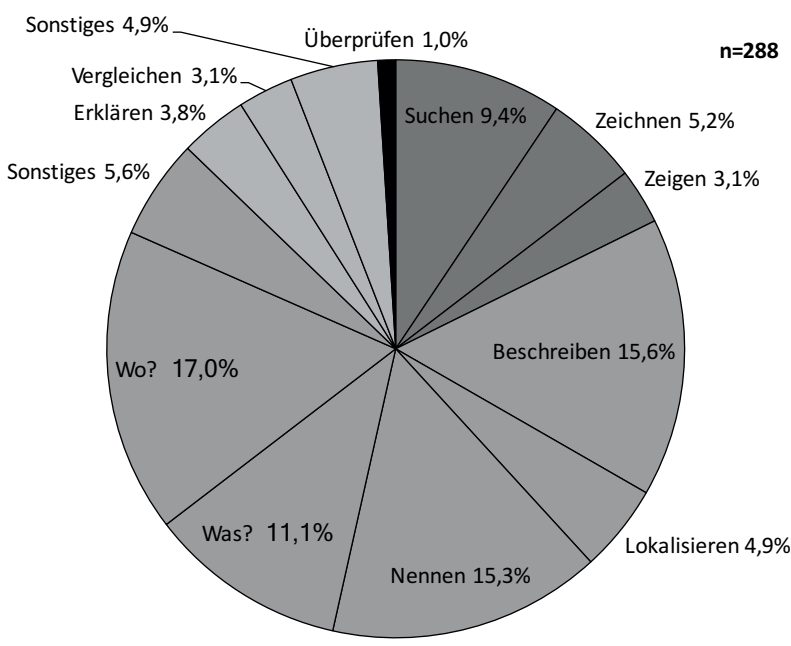

Abb. 4: Verwendete Operatoren und Fragewörter bei der Kartenarbeit. Die Gesamtzahl $n=288$ entspricht der Anzahl der Operatoren und Fragewörter bei kartenbezogenen Aufgabenstellungen, die klar einem der Anforderungsbereiche zugeordnet werden konnten ( $G A=$ Grundanforderungsbereich, $A F B=$ Anforderungsbereich). Unter, Sonstiges' fallen Operatoren, deren Anteil unter zwei Prozent liegt (eigene Darstellung).

2 Es wurde von Lindau (2012) nicht in Grundanforderungsbereich und Anforderungsbereich I unterschieden.
Kartenarbeit im Rahmen ihres Unterrichts fördern. Die Unterrichtsbeobachtungen zeigen, dass nur bei rund $36 \%$ aller im Unterricht behandelten Karten eine sprachliche Förderung durch die Lehrkräfte stattfindet. Bei $64 \%$ der behandelten Karten findet keinerlei Förderung statt.

Es wurde erwartet, dass die sprachlichen Fördermaßnahmen der Lehrkräfte mit steigender Klassenstufe der Schüler/innen abnehmen würden, da zu erwarten ist, dass deren sprachliche Fähigkeiten ansteigen. Es zeigte sich jedoch kein klares Muster. Besonders häufig kamen sprachliche Fördermaßnahmen nicht in der 5./6., sondern in der 7./8. und in der 10./11. Jahrgangsstufe vor. Dies könnte daran liegen, dass die sprachlichen Anforderungen mit steigender Klassenstufe zunehmen und die Lehrkräfte darauf mit zusätzlichen sprachlichen Hilfen reagieren. Die häufigste Fördermaßnahme ist die "Umformulierung der Schüler/innenantwort" durch die Lehrkräfte (siehe Abb. 5). Diese Fördermaßnahme wird häufiger von älteren Lehrkräften als von jüngeren Lehrer/innen eingesetzt (siehe Abb. 6). Möglicherweise legen ältere Lehrkräfte ihrem Unterricht stärkere bildungssprachliche Normen zugrunde, deren Einhaltung sie durch Umformulierungen der Schüler/innenäußerungen deutlich machen als jüngere Lehrkräfte. Dabei ist allerdings nicht klar, ob die Umformulierungen von den Lehrkräften bewusst zur sprachlichen Förderung der Schüler/innen durch das Geben sprachlicher Vorbilder eingesetzt werden, oder ob die Lehrer/innen lediglich das Unterrichtsgespräch vorantreiben wollen und auf die inhaltlich relevanten Aspekte der Schüler/innenantwort eingehen.

Ebenfalls bedeutsam ist die Aufforderung der Lehrkräfte an die Schüler/innen, ihre Antworten zu präzi-

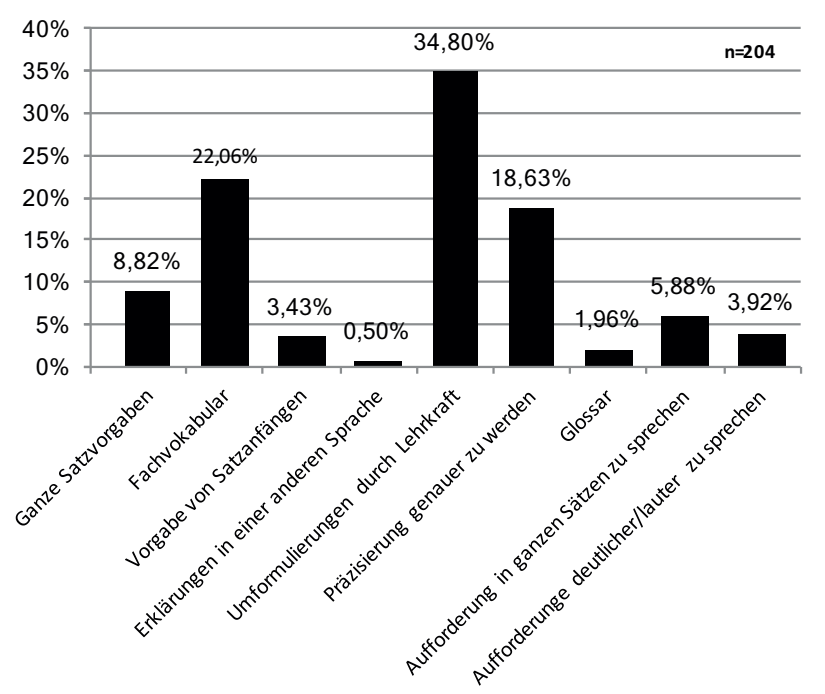

Abb. 5: Maßnahmen der Lehrkräfte zur sprachlichen Förderung der Schüler/innen bei der Kartenarbeit. Die Gesamtzahl n=204 entspricht der Anzahl der genutzten sprachlichen Hilfen bei der Kartenarbeit (eigene Darstellung) 


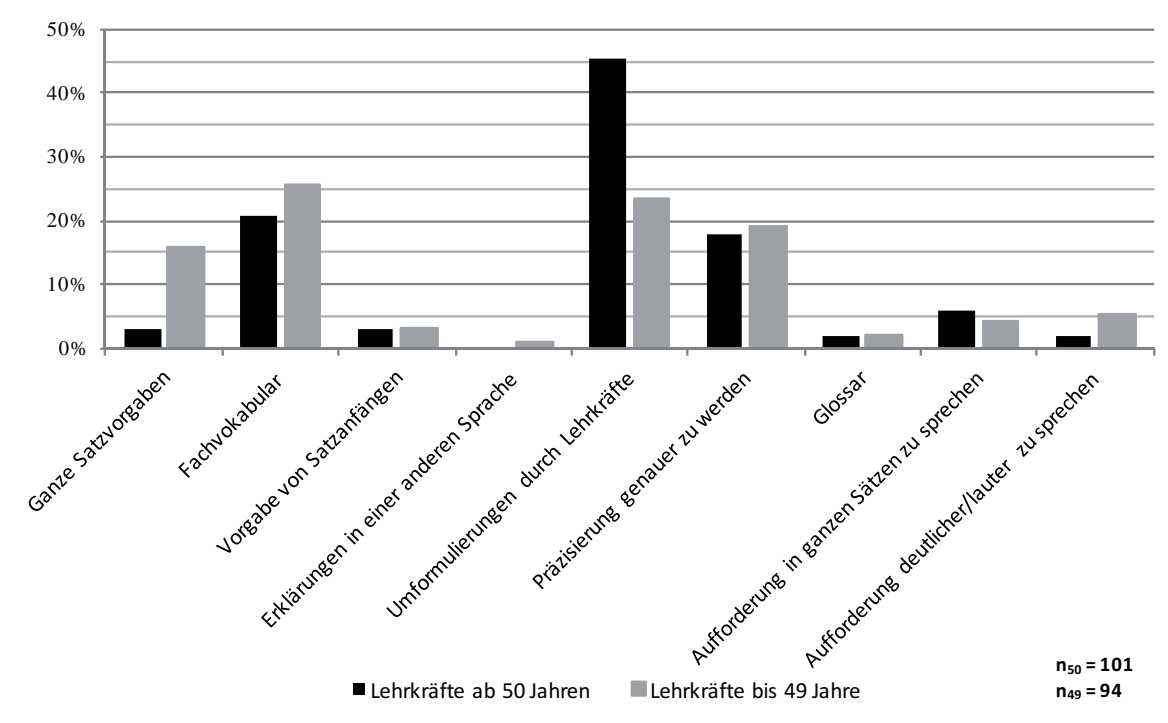

Abb. 6: Sprachliche Fördermaßnahmen bei der Kartenarbeit im Vergleich von älteren (ab 50 Jahren) und jüngeren Lehrkräften (bis 49 Jahre). Die Gesamtzahl n50 = 101 entspricht der Anzahl der beobachteten Fördermaßnahmen durch Lehrkräfte ab einem Alter von 50 Jahren, $n 49=94$ bezieht sich auf die Anzahl der beobachteten Fördermaßnahmen durch Lehrkräfte bis 49 Jahre. Bei neun Personen wurde das Alter nicht erhoben und deren sprachliche Fördermaßnahmen wurden hier nicht berücksichtigt (eigene Darstellung). sieren. Es handelt sich hier um spontane Reaktionen der Lehrkräfte im Unterrichtsgespräch auf die Äußerungen der Schüler/innen. Vermutlich versuchen die Lehrer/innen auf diese Weise sowohl die inhaltliche als auch die sprachliche Qualität der Schüler/innenäußerungen zu verbessern, die allgemeine Verständlichkeit der Schüler/innenkommentare zu erhöhen und positive Vorbilder zu schaffen. Zudem ist erkennbar, dass die Lehrkräfte versuchen, das Fachvokabular der Schüler/innen zu erweitern. Es werden häufig Fachwörter bereitgestellt und es wird auf deren korrekte Verwendung durch die Schüler/innen geachtet (siehe Abb. 5). Die Vermittlung von Fachvokabular scheint jüngeren Lehrkräften etwas wichtiger zu sein als älteren (siehe Abb. 6).

Es fällt auf, dass es sich bei den beobachteten sprachlichen Fördermaßnahmen bei der Kartenarbeit vorrangig um spontane Reaktionen der Lehrkräfte im Unterrichtsgespräch handelt. Geplante und durch Unterrichtsvorbereitungen gezielt eingesetzte Förderungen wie z. B. das Scaffolding (z. B. die Vorgabe von Satzanfängen) oder das ,Glossar wurden nur sehr selten beobachtet. Entsprechend der geringen Bedeutung von Aufgaben im Anforderungsbereich III wurden sprachliche Hilfen bei der Vorbereitung und Formulierung von kartenbasierten Argumentationen, Erörterungen, Diskussionen und Erklärungen nicht beobachtet. Zudem wurden im Bereich der sprachlichen Förderung keinerlei Differenzierungsmaßnahmen realisiert, durch welche sprachlich schwächere Schüler/innen gezielt hätten unterstützt werden können. Auch auf den Migrationshintergrund und die verschiedenen fremdsprachlichen Kompetenzen wurde in dem von uns beobachteten Unterricht nur sehr selten eingegangen. Lediglich einmal hat eine Lehrkraft auf ihre Fremdsprachenkompetenz zurückgegriffen, um einer Person etwas zu erklären (siehe Abb. 6).

\section{Diskussion und Fazit}

Die Unterrichtsbeobachtungen belegen eindrucksvoll die hohe quantitative Bedeutung von Karten im Geographieunterricht, da sie in über der Hälfte der Stunden eingesetzt wurden.

Die geringe Berücksichtigung von Aufgaben im Anforderungsbereich III und die geringe Bedeutung gezielter Sprachförderung deuten jedoch darauf hin, dass sowohl die inhaltliche als auch die sprachliche Qualität bei der Kartenarbeit im Geographieunterricht noch erhöht werden könnte. Die Funktion von Karten im Rahmen gesellschaftlicher Diskurse, zum Verständnis von Argumentationen und zur Begründung eigener Beurteilungen steht bisher offensichtlich nicht im Fokus des Unterrichts. Damit können die in den Lehrplänen vorgesehenen anspruchsvollen geographischen Fragestellungen nur in Ansätzen beantwortet werden und das Potential des Faches zur Entwicklung von mündigen Bürgerinnen und Bürgern, die über umfangreiche Kartenkompetenzen verfügen, wird nicht voll ausgeschöpft. Mögliche Gründe könnten u.a. die fehlenden Kompetenzen der Lehrkräfte sein. Tatsächlich zeigt eine Studie von Uhlenwinkel (2015), dass Geographiestudierende oftmals große Schwierigkeiten haben, eigene qualitätsvolle (kartenbasierte) Argumentationen zu entwickeln. Wenn diese Kompetenzen allerdings nicht im Laufe des Studiums erworben werden, ist nicht zu erwarten, dass Lehrkräfte die Argumentationskompetenzen der Schüler/innen diagnostizieren und Maßnahmen für einen sprachbewussten Geographieunterricht umsetzen können. Eine Untersuchung von Abiturklausuren zeigt demnach, dass die Lehrer/innen größere Schwierigkeiten bei der Bewertung von Schülerargumentationen haben (vgl. Gohrbandt et al. 2013). Ein weiterer Grund für das geringe Vorkommen von Aufgaben im Anforderungsbereich III könnte auch darin liegen, 
dass die Lehrkräfte deren Bedeutung nicht erkennen. Interviews mit Geographielehrkräften zum Thema der Argumentation deuten auf diesen Zusammenhang hin (vgl. Budke 2012a). Möglicherweise wird die Förderung von fachbezogenen Sprachkenntnissen ebenso wenig als Voraussetzung für intensive inhaltliche Auseinandersetzungen gesehen. Dies scheint eng mit dem Fachverständnis einiger Befragten verbunden zu sein, die in ihrem Unterricht vor allem Wissen und geographische Konzepte vermitteln wollen und in diesem Kontext die Relevanz von Argumentationen und Sprachförderung nicht sehen. Zudem hatten einige Befragte sehr wenig Wissen und Erfahrung darüber, wie man Argumentationskompetenzen bei den Schülerinnen und Schülern gezielt aufbauen und fördern könnte (vgl. ebd.). Hinzu kommen die teilweise negativen Erfahrungen vieler Befragter mit Argumentationen in ihrem Unterricht, was dazu führt, dass diese zukünftig nur in geringem Umfang zugelassen werden und hauptsächlich dann, wenn sie sich spontan im Unterricht ergeben. Ein weiterer Ursachenkomplex lässt sich den teilweise negativen Rahmenbedingungen zuordnen. Geographieunterricht wird in der Sekundarstufe I in der Regel nur einstündig unterrichtet, wodurch die Behandlung komplexer Fragestellungen, welche aus verschiedenen Perspektiven erörtert und bewertet werden müssen, erschwert wird. Lehrer/innen, die zur Politischen Bildung im Geographieunterricht befragt wurden, gaben häufig an, dass die ,Stofffülle dazu führe, dass die Themen teilweise nur oberflächlich behandelt würden (vgl. Budke et al. 2016). Zudem liegt noch zu wenig Unterrichtsmaterial vor, welches zur fachspezifischen Förderung von Sprach- und Argumentationskompetenzen eingesetzt werden kann. Die Lehrer/innen müssen demnach aufwendige Entwicklungsarbeit leisten, wenn sie diese Kompetenzen dennoch bei den Schülerinnen und Schülern fördern möchten.

Aus diesen Überlegungen lässt sich folgern, dass die Integration von Sprachförderung insbesondere mit dem Fokus auf Argumentieren in die universitäre Lehrer/innenausbildung eine wichtige Voraussetzung für die Entwicklung des sprachbewussten Geographieunterrichts darstellt. Dabei müssen zunächst die Sprachkompetenzen der Studierenden verbessert werden und ihnen dann didaktische Möglichkeiten zur gezielten Förderung aufgezeigt werden. Dies betrifft auch die sprachliche Förderung von Kindern mit sonderpädagogischem Förderbedarf sowie die Förderung von (neu zugewanderten) Kindern, die Deutsch als Fremdsprache lernen. Dabei müssen die Ansätze, die im Bereich Deutsch als Zweitsprache (DaZ) entwickelt wurden, auf die Geographie bezogen werden.

Ein weiteres wichtiges Handlungsfeld ist die Weiterentwicklung der Schulbücher und die Entwicklung von didaktischen Materialien zur Sprachförderung. Einige Ansätze zur Förderung von Argumentationskompetenzen finden sich bei Budke (2012b). Eine Möglichkeit besteht darin, die Sprachförderansätze, welche bereits im Bereich des bilingualen Geographieunterrichts erfolgreich erprobt wurden, auf den Geographieunterricht in deutscher Sprache zu übertragen. Letztlich müssen noch weitere empirische Untersuchungen stattfinden, um die Realisierung eines sprachbewussten Geographieunterrichts noch detaillierter erfassen zu können.

\section{Acknowledgement}

We acknowledge support by Deutsche Forschungsgemeinschaft (DFG) and Open Access Publishing Fund of Osnabrück University.

\section{$7 \quad$ Literatur}

Bauriedl, S. (2007): Räume lesen lernen: Methoden zur Raumanalyse in der Diskursforschung. In: Forum: Qualitative Sozialforschung/Social Research 8/2. http:// www.qualitative-research.net/index.php/fqs/article/ view/236/524 (02.10.2016).

Becker-Mrotzek, M., K. Schramm, E. Thürmann \& H. J. Vollmer (2013): Sprache im Fach. Einleitung. In: ebd. (Hrsg.): Sprache im Fach. Sprachlichkeit und fachliches Lernen. (= Fachdidaktische Forschungen 3), Berlin: Waxmann. S. 7-24.

Bortz, J. \& Döring, N. (2015): Forschungsmethoden und Evaluation für Human- und Sozialwissenschaften. Berlin: Springer.

Budke, A. (2011): Förderung von Argumentationskompetenzen in aktuellen Geographieschulbüchern. In: Matthes, E. \& C. Heinze (Hrsg.): Aufgaben im Schulbuch. Bad Heilbrunn. S. 253-264.

Budke, A. (2012a): Argumentationen im Geographieunterricht. In: Geographie und ihre Didaktik/ Journal ofGeography Education 40/1. S. 23-34.

Budke, A. (Hrsg.; 2012b): Diercke Kommunikation und Argumentation. Braunschweig: Westermann.

Budke, A. \& G. Weiss (2014): Sprachsensibler Geographieunterricht. In: Michalak, M. (Hrsg.): Sprache als Lernmedium im Fachunterricht. Theorien und Modelle für das sprachbewusste Lehren und Lernen. Baltmannsweiler: Schneider Verlag Hohengehren. S. 113-133.

Budke, A., M. Kuckuck, M. Michalak \& B. Müller (2016): Erstellung kartenbasierter Argumentationen - Strukturierungs- und Formulierungshilfen. In: Praxis Geographie 46 (6), 46-48.

CEFR - Council of Europe 2011. CEFR - Common European Framework of Reference for Languages. https:// www.coe.int/t/dg4/linguistic/source/framework_en.pdf (13.10.2016).

DGfG: Deutsche Gesellschaft für Geographie (Hrsg.; 2014): Bildungsstandards im Fach Geographie für den 
Mittleren Bildungsabschluss mit Aufgabenbeispielen. Bonn: Selbstverlag DGfG.

Diegmann, D. (2013): Die Beobachtung. In: Drinck, B. (Hrsg.): Forschen in der Schule. Ein Lehrbuch für (angehende) Lehrerinnen und Lehrer. Stuttgart: Budrich. S. 182-226.

Foucault, M. (1973). Die Archäologie des Wissens. Frankfurt am Main: Suhrkamp.

Gogolin, I., I. Dirim, T. Klinger, I. Lange, D. Lengyel, U. Michel, U. Neumann, H. H. Reich, H. J. Roth \& K. Schwipper (2011): Förderung von Kindern und Jugendlichen mit Migrationshintergrund. FÖRMIG Bilanz und Perspektiven eines Modellprogramms. Münster/New York: Waxmann.

Gohrbandt, E., J. Mäsgen, G. Weiss \& D. Wiktorin (2013): Zwischen Materialschlacht und Reproduktion: Schriftliche Zentralabituraufgaben Geographie im Bundesländervergleich und erste Erkenntnisse zu Schülerleistungen. In: Geographie und Schule 35/206. S. 4-14.

Gryl, I. \& D. Kanwischer (2011): Geomedien und Kompetenzentwicklung - ein Modell zur reflexiven Kartenarbeit im Unterricht. In: Zeitschrift für Didaktik der Naturwissenschaften 17. S. 177-202.

Gryl, I., M. Horn, K. Schweizer, D. Kanwischer \& T. Rhode-Jüchtern (2010): Reflexion und Metaperspektive als notwendige Komponenten der Kartenkompetenz. In: Geographie und ihre Didaktik 38/3. S. 172-179.

Hemmer, M., I. Hemmer, A. Hüttermann \& M. Ullrich (2010): Kartenauswertekompetenz - Theoretische Grundlagen und Entwurf eines Kompetenzstrukturmodells. In: Geographie und ihre Didaktik 38/3. S. 158-171.

Kniffka, G.M. \& B.S. Neuer (2008): „Wo geht's hier nach Aldi?“ - Fachsprachen lernen im kulturell heterogenen Klassenzimmer. In: Budke, A. (Hrsg.): Interkulturelles Lernen im Geographieunterricht. Potsdam: Univ.-Verlag. S. 121-135.

Kölzer, C., V. Lemke \& M. Michalak (2015): Diagramme im gesellschaftswissenschaftlichen Unterricht - eine Herausforderung für Lernende mit Deutsch als Zweitsprache. In: Zeitschrift für Didaktik der Gesellschaftswissenschaften 6/2. S. 121-135.

Lenz, T. (2005): Thematische Karten im Geographieunterricht. In: Geographie heute 26/229. S. 2-9.

Lindau, A. (2012): Der Kompetenzbereich „Räumliche Orientierung“ im Geographieunterricht - Ergebnisse einer Beobachtungsstudie. In: Hüttermann, A., P. Kirchner, S. Schuler \& K. Drieling (Hrsg.): Räumliche Orientierung. Räumliche Orientierung, Karten und Geoinformation im Unterricht. Geographiedidaktische Forschungen 49, Braunschweig: Westermann. S. 42-53.

Mattisek, A., C. Pfaffenbach \&P. Reuber (2013): Methoden der empirischen Humangeographie. Braunschweig: Westermann.

Michalak, M., V. Lemke \& M. Goeke (2015): Sprache im Fachunterricht: Eine Einführung in Deutsch als Zweitsprache und sprachbewussten Unterricht. Tübingen: NarrFranckeAttempto.

Morawski, M. \& Budke, A. (2017a). Language Awareness in Geography Education - An Analysis of the Potential of
Bilingual Geography Education for Teaching Geography to Language Learners. In: European Journal of Geography: Special Issue EUROGEO 20167/5. S. 61-85.

Mose, J. \& A. Strüver (2009): Diskursivität von Karten - Karten im Diskurs. In: Glasze, G. \& A. Mattisek (Hrsg.): Handbuch Diskurs und Raum. Theorien und Methoden für die Humangeographie sowie die sozial- und kulturwissenschaftliche Raumforschung. Bielefeld: transcript. S. 315-325.

Plepis, M. (2013): Strategien von Schülerinnen und Schülern zur Auswertung komplexer thematischer Karten Eine empirische Untersuchung in der Jahrgangsstufe 10 der Realschule (= Münsteraner Arbeiten zur Geographiedidaktik 5), Münster: Univ.-Verlag.

Roos, M. \& B. Leutwyler (2011): Wissenschaftliches Arbeiten im Lehramtsstudium. Bern: Huber.

Schnell, R., P.B. Hill \& E. Esser (2014): Methoden der empirischen Sozialforschung. München: Oldenbourg.

Schnotz, W. (2002): Wissenserwerb mit Texten, Bildern und Diagrammen. In: Issing, L. J. \& P. Klimsa (Hrsg.): Information und Lernen mit Multimedia und Internet. Lehrbuch für Studium und Praxis. Weinheim: Beltz. S. 64-81.

Schnotz, W. \& S. Dutke (2004): Kognitionspsychologische Grundlagen der Lesekompetenz: Mehrebenenverarbeitung anhand multipler Informationsquellen. In: Schiefele, U., C. Artelt, W. Schneider \& P. Stanat (Hrsg.): Struktur, Entwicklung und Förderung von Lesekompetenz. Vertiefende Analysen im Rahmen von PISA 2000. Wiesbaden: VS Verlag für Sozialwissenschaften. S. 61-99.

Uhlenwinkel, A. (2015): Geographisches Wissen und geographische Argumentation. In: Budke, A., M. Kuckuck, M. Meyer, F. Schäbitz, K. Schlüter \& G. Weiss (Hrsg.): Fachlich argumentieren lernen. Didaktische Forschungen zur Argumentation in den Unterrichtsfächern (=LehrerInnenbildung gestalten 7), Münster: Waxmann. S. 46-61.

Ullrich, M., W. Schnotz, H. Horz, N. McElvany, S. Schroeder \& J. Baumert (2012): Kognitionspsychologische Aspekte eines Kompetenzmodells zur Bild-Text-Integration. In: Psychologische Rundschau 63/1. S. 11-17.

Vollmer, H. J. (2011): Schulsprachliche Kompetenzen: Zentrale Diskursfunktionen. http://www.home.uni-osnabrueck.de/hvollmer/VollmerDF-Kurzdefinitionen.pdf (03.10.2016).

Vollmer, H. J. \& E. Thürmann (2013): Sprachbildung und Bildungssprache als Aufgabe aller Fächer der Regelschule. In: Becker-Mrotzek, M., K. Schramm, E. Thürmann \& H. J. Vollmer (Hrsg.): Sprache im Fach. Sprachlichkeit und fachliches Lernen (= Fachdidaktische Forschungen 3), Berlin: Waxmann. S. 41-58.

Weidenmann, B. (2004): Psychologische Ansätze zur Optimierung des Wissenserwerbs mit Bildern. In: SachsHombach, K.\& K. Rehkämper (Hrsg.): Bild - Bildwahrnehmung - Bildverarbeitung. Interdisziplinäre Beiträge zur Bildwissenschaft. Wiesbaden: Deutscher Universitätsverlag. S. 243-254.

Werlen, B. (1997): Sozialgeographie alltäglicher Regionalisierungen: Globalisierung, Region und Regionalisierung (= Erdkundliches Wissen 119), Stuttgart: Steiner.

Wiegand, P. (2006): Learning and teaching with maps. London: Routledge. 